\title{
ON THE DIFFERENTIAL FORMS ON ALGEBRAIC VARIETIES
}

\author{
YÛSAKU KAWAHARA
}

Introduction. In the book "Foundations of algebraic geometry" ${ }^{1)} \mathrm{A}$. Weil proposed the following problem; does every differential form of the first kind on a complete variety $\mathbf{U}$ determine on every subvariety $\mathbf{V}$ of $\mathbf{U}$ a differential form of the first kind? This problem was solved affirmatively by S. Koizumi when $\mathbf{U}$ is a complete variety without multiple point.' ${ }^{2)}$ In this note we answer this problem in affirmative in the case where $\mathbf{V}$ is a simple subvariety of a complete variety $\mathbf{U}$ (in $\S 1$ ). When the characteristic is 0 we may extend our result to a more general case but this does not hold for the case characteristic $p \neq 0$ (in $\S 2$ ).

I express my hearty thanks to Prof. Y. Akizuki and Mr. S. Koizumi for their useful remarks.

$\S 1$. Let $K=k\left(x_{1}, \ldots, x_{N}\right)=k(x)$ be a field, generated over a field $k$ by a set of quantities $(x)$, the class $\mathfrak{P}$ of equivalent $(n-1)$-dimensional valuations for $K / k$ is called a prime divisor in the sense of Zariski, ${ }^{3)} n$ being the dimension of $K$ over $k$, and its normalized valuation with rational integers as the value group is denoted by $\nu_{\mathfrak{\beta}}$. Let $F(x, d x)$ be a differential form belonging to the extension $k(x)$ of $k$. We say that $F(x, d x)$ is finite at $\mathfrak{P}$ if $F(x, d x)$ is of the form

$$
F(x, d x)=\sum z_{\alpha_{\beta}} \ldots d y_{\alpha} d y_{\beta} \ldots,
$$

where $\nu_{\mathfrak{F}}\left(z_{\alpha \beta} \ldots\right) \geq 0, \nu_{\Re}\left(y_{\alpha}\right) \geqslant 0, \nu_{\mathfrak{\beta}}\left(y_{\beta}\right) \geqslant 0, \ldots$.

THEOREM 1. Let $\mathbf{U}^{n}$ be a complete variety and $k$ a field of definition of $\mathbf{U}^{n}$ which is perfect. Let $\mathbf{P}$ be a generic point of $\mathbf{U}^{n}$ over $k$. Then, for every differential form $\omega$ on $\mathbf{U}$ defined over $k, \omega(\mathbf{P})$ is of the first kind if and only if it is finite at every prime divisor of $k(\mathbf{P})$.

Proof. Sufficiency. Let $(y)$ be a set of quantities such that $k(\mathbf{P})=k(y)$ and let $P^{\prime}$ be a simple point of the locus $V^{n}$ of $(y)$ over $k$. If $P^{*}$ is a generic

Received June 19, 1951.

1) We refer this book by $F$ in this note.

2) S. Koizumi, On the differential forms of the first kind on algebraic varieties. I. Journal of the Mathematical Society of Japan, vol. 1 (1949). II. vol. 2 (1951).

3) See $\mathrm{O}$. Zariski, The reduction of the singularities of an algebraic surface. Annals of Math. vol. 40 (1939). 
point of any $(n-1)$-dimensional simple subvariety of $V^{n}$ over the algebraic closure $\bar{k}$ of $k$, then $\omega(\mathbf{P})$ is finite at $P^{*}$ by our hypothesis. Therefore by Prop. 5 in Koizumi's paper ${ }^{4)} \omega(\mathbf{P})$ is finite at $P^{\prime}$, which shows that $\omega(\mathbf{P})$ is of the first kind.

Necessity. There exists a set of quantities $(y)$ such that $k(\mathbf{P})=k(y)$ and that, on the locus $V$ of $(y)$ over $k$, the center of $\mathfrak{B}$ is an $(n-1)$-dimensional simple subvariety $W . \quad V$ is obtained by a birational transformation such that the center of $\mathfrak{F}$ is an $(n-1)$-dimensional subvariety and by the normalization over $k$ of the resulting variety. Let $P^{\prime}$ be a generic point of $W$ over $k$ and let $\left(t_{1}, \ldots, t_{n}\right)$ be a set of uniformizing parameters in $k(y)$ for $V$ at $P^{\prime}$. Since $\omega(\mathbf{P})$ is of the first kind,

$$
\omega(\mathbf{P})=\sum w_{i j} \ldots d t_{i} d t_{j} \ldots,
$$

where $w_{i j} \ldots$ are in the specialization ring of $P^{\prime}$ in $k(y)=k(\mathbf{P})$. As $t_{1} \ldots, t_{n}$ are in the specialization ring of $P^{\prime}$ in $k(y)$ and this specialization ring is identical with the valuation ring of $\mathfrak{B}$, the theorem is proved.

Remark. This theorem holds without the assumption that $k$ is a perfect field if each $\mathfrak{P}$ can be uniformized under a birational transformation of $\mathbf{U}$ over $k$, a fortiori, if $\mathrm{U}$ has no singular point.

The set of elements $\left(t_{1}, \ldots, t_{n}\right)$ in the proof (necessity) of th. 1 is called $a$ set of uniformizing parameters at $\mathfrak{P}$. $A$ differential form is finite at $\mathfrak{P}$ if and only if it is expressed in one and only one way as a polynomial in $d t_{1}, \ldots, d t_{n}$ with coefficients in the valuation ring of $\mathfrak{B}$.

LemMa 1. Let $\mathbf{U}^{n}$ be a variety defined over $k$ and let $\mathbf{V}^{m}$ be a simple subvariety of $\mathrm{U}^{n} \cdot$ which is algebraic over $k$. Then there exists a series of algebraic varieties

$$
\mathbf{U}^{n}=\mathbf{U}_{0}^{n}, \mathbf{U}_{1}^{n-1}, \mathbf{U}_{2}^{n-2}, \ldots, \mathbf{U}_{n-m}^{m}=\mathbf{V}^{m}
$$

such that each $\mathbf{U}_{i}$ is algebraic over $k$ and that $\mathbf{U}_{i+1}$ is a simple subvariety of $\mathbf{U}_{i}$ $(i=0, \ldots, n-m-1)$.

Proof. Since it is enough to prove this for affine varieties, we may assume that $U^{n}$ is contained in affine $N$-space $S^{N}$. Let $P=(y)$ be a generic point of $V^{m}$ over $\bar{k}_{.}$As $P$ is a simple point of $U^{n}, U^{n}$ is defined by a set of equations $F_{\mu}(X)=0$, where $F_{\mu}(X)$ are polynomials in $k\left[X_{1}, \ldots, X_{N}\right]$ and the rank of the Jacobian matrix $\left\|\partial F_{\mu} / \partial y_{i}\right\|$ is $N-n$. Further as $P$ is a generic point of $V^{m}, V^{m}$ is defined by a set of equations $G_{\downarrow}(X)=0$, where $G_{\downarrow}(X)$ are polynomials in $\bar{k}\left[X_{1}, \ldots, X_{N}\right]$ and the rank of the matrix $\left\|\partial G_{\nu} / \partial y_{i}\right\|$ is $N-m$. Since we may assume $n>m$, there must exist a $\nu$ such that the rank of the matrix $\left|\begin{array}{l}\partial F_{\mu} / \partial y_{i} \\ \partial G_{\nu} / \partial y_{i}\end{array}\right|$ is $N-n+1$; we may assume without loss of generality that

4) Loc. cit. "). 
$\nu=1$. Further we may assume that $G_{1}(X)$ is irreducible. Let $W^{x-1}$ be the variety defined by $G_{1}(X)=0$ in $S^{N}$. There exists a component $U_{1}$ of the intersection of $W^{N-1}$ and $U^{n}$ which contains $V^{m}$ (F. IV $V_{4}$ th. 8). The dimension of $U_{1}$ is $n-1$ (F. VI th. 1 Cor. 2) and by the construction it is obvious that $V^{m}$ is a simple subvariety of $U^{n-1}$. Thus our assertion follows by induction on $n$.

Lemma 2. Let $k$ be a perfect field and let $P=(x)$ be a set of quantities such that $k(P)$ is a regular n-dimensional extension of $k$. Let $v$ be an $(n-2)$-dimensional valuation of $k(P)$ of rank $2^{5)}$ Then there exists a variety $U^{n}$ defined over $k$ with a generic point $Q$ such that $k(P)=k(Q)$ and that the center of the valuation $v$ on $U$ is a simple subvariety $V^{n-2}$ of $U$.

Proof. Let $\mathfrak{D}$ be the valuation ring of $v$ and let $m$ be the prime ideal of all the non-units in $\mathfrak{D}$. By our hypothesis, the residue class field $\mathfrak{D} / \mathrm{m}$ is $(n-2)$ dimensional over $k$. Let $\left(u_{1}, \ldots, u_{n-2}\right)$ be a system of elements in $D$ such that they are algebraically independent $\bmod m$ over $k$. Put $k\left(u_{1}, \ldots, u_{n-2}\right)=K$. Then $k(P)$ is 2-dimensional over $K$. We can also select $\left(u_{1}, \ldots, u_{n-2}\right)$ in such a way that $k(P)$ is separably generated over $K$. As $v(z)=0$ for each element $z \neq 0$ in $K$, we can consider $v$ as a valuation of dimension 0 and rank 2 of $k(P) / K$. By Zariski's local uniformization theorem (cf. O. Zariski, Reduction of algebraic three-dimensional varieties $\S \S 10-12, \S 16){ }^{6}$ ) there exists such a set of quantities $\left(y_{1}, \ldots, y_{n}\right)$ that $k(P)=K(y)$ and that the quotient ring $\mathfrak{D}_{\bar{p}}$ of $\vec{p}=K[y] \cap m$ in $K[y]$ is a regular local ring. Put $Q=\left(u_{1}, \ldots, u_{n-2}, y_{1}, \ldots, y_{m}\right)$ and let $U$ be its locus over $k$. The quotient ring $\mathfrak{O}_{\mathfrak{p}}$ of $\mathfrak{p}=k\left[u_{1}, \ldots, u_{n-2}, y_{1}, \ldots, y_{m}\right] \cap m$ in $k\left[u_{1}, \ldots, u_{n-2}, y_{1}, \ldots, y_{m}\right]$ is identical with $\mathfrak{D}_{\mathfrak{p}}$ and hence it is also regular local ring. As $k$ is perfect, $\mathfrak{p}$ defines in $U$ absolutely simple subvariety in the sense of Zariski. Hence there exists a simple point $Q^{\prime}$ of $U$ whose specialization ring in $k(Q)$ is identical with $\Sigma_{p}$.

THEOREM 2. Let $U^{n}$ be a complete variety and $\mathbf{V}$ its simple subvariety. If a differential form $\omega$ on $\mathrm{U}$ is of the first kind, then it induces on $\mathrm{V}$ a differential form $\omega^{\prime}$ of the first kind.

Proof. It is known that a differential form which is finite on $\mathbf{V}$ induces uniquely a differential form $\omega^{\prime}$ on $V^{7} .^{\prime}$ We prove that this $\omega^{\prime}$ is of the first kind. We may assume that $\mathbf{U}, \mathbf{V}$ and $\omega$ have a common field of definition $k$ which is perfect. Let $\mathbf{P}$ be a generic point of $\boldsymbol{U}$ over $k$ and let $\mathbf{Q}$ be a generic point of $\mathbf{V}$ over $k$. By lemma 1 we may assume without loss of generality that the dimension of $\mathbf{V}$ is $n-1$. Let $\mathfrak{B}^{\prime}$ be a prime divisor of $k(\mathbf{Q})\left(\nu_{\mathfrak{P}^{\prime}}\right.$ being a $(n-2)$ -

5) Loc. cit. ${ }^{3)}$ 。

6) O. Zariski, Reduction of singularities of algebraic three-dimensional varieties, Annals of Math. vol. 45 (1944).

i) Loc. ict 2) S. Koizumi I. Prop. 6. 
dimensional valuation over $k$ ). We shall prove that $\omega^{\prime}(\mathbf{Q})$ is finite at $\mathfrak{P}^{\prime}$. As $\mathbf{Q}$ is a simple point of $\mathbf{U}$ of dimension $n-1$ over $k$, it determines a prime divisor $\mathfrak{P}$ in $k(\mathbf{P})$; namely the valuation ring of $\mathfrak{P}$ is identical with the specialization ring of $\mathbf{Q}$ in $k(\mathbf{P})$. We may construct, by virtue of $\mathfrak{P}$ and the prime divisor $\mathfrak{P}^{\prime}$ of $k(\mathbf{Q})$, a valuation $v$ of dimention $n-2$, and rank 2 of $k(P)$. It follows from lemma 2 that there exists a variety $U^{\prime n}$ and a point $Q^{\prime}$ of $U^{\prime}$ such that $Q^{\prime}$ is simple on $U^{\prime}$ and the specialization ring of $Q^{\prime}$ is contained in the valuation ring of the valuation $v$ of $k(\mathbf{P})$. Let $\left(t_{1}, \ldots, t_{n}\right)$ be a system of uniformizing parameters of $Q^{\prime}$ in $k(\mathbf{P})$. Since $\omega$ is of the first kind $\omega(\mathbf{P})$ is of the form

$$
\omega(\mathbf{P})=\sum w_{i j} \ldots d t_{i} d t_{j} \ldots
$$

where $w_{i j} \ldots, t_{i}, t_{j}$, etc. are contained in the specialization ring of $Q^{\prime}$; therefore $v\left(w_{i j} \ldots\right) \geqslant 0, v\left(t_{i}\right) \geqslant 0, \ldots$ and $\nu_{\mathfrak{P}}\left(w_{i j} \ldots\right) \geqslant 0, \nu_{\mathfrak{P}}\left(t_{i}\right) \geqslant 0$; namely $w_{i j} \ldots, t_{i}, \ldots$ are contained in the specialization ring of $\mathbf{Q}$ in $k(\mathbf{P})$. Therefore the specializations of $w_{i j} \ldots, t_{i}, t_{j}$ over $\mathbf{P} \rightarrow \mathbf{Q}$ with respect to $k$ are contained in the valuation ring of $\mathfrak{P}^{\prime}$ in $k(\mathbf{Q})$. This proves that $\omega^{\prime}(\mathbf{Q})$ is finite at $\mathfrak{P}^{\prime}$.

\section{The case of characteristic 0 .}

Let $\mathbf{U}^{n}$ be a complete variety defined over $k$ with a generic point $\mathbf{P}$ over $k$ and let $\mathbf{V}$ be its subvariety defined over $k$ with a generic point $\mathbf{Q}$ over $k$. If a differential form $\omega$ has the following expression

$$
\omega(\mathbf{P})=\sum z_{\alpha_{3}} \ldots d y_{\alpha} d y_{\beta} \ldots,
$$

where $z_{\alpha_{\beta}} \ldots, y_{\alpha}, y_{\beta}, \ldots$ are contained in the specialization ring of $\mathbf{Q}$ in $k(\mathbf{P})^{8)}$ then we can induce $\omega$ on $\mathbf{V}$ even if $\mathbf{Q}$ is not a simple point of $\mathbf{U}$.

In this section we assume that the characteristic is 0 and prove that if $\omega$ is a differential form of the first kind on $\mathbf{U}$ it induces uniquely on $\mathbf{V}$ a differential form $\omega^{\prime}$ of the first kind.

THEOREN 3. If a differential form $\omega(\mathbf{P})=\sum z_{\alpha \beta} \ldots d y_{\alpha} d y_{\beta} \ldots$ is finite at $\mathbf{Q}$, then $\omega^{\prime}(Q)=\sum z_{\alpha \beta}^{\prime} \ldots d y_{\alpha}^{\prime} d_{\beta}^{\prime} \ldots$ is uniquely determined by $\omega(\mathbf{P})$, where $z_{\alpha \beta}^{\prime} \ldots, y_{\alpha}^{\prime}$, $y_{\beta}^{\prime}$ are the specializations of $z_{\alpha_{\beta}} \ldots, y_{\alpha}, y_{\beta}$, over $\mathbf{P} \rightarrow \mathbf{Q}$ with respect to $k$.

Proof. We prove that if $\omega(\mathbf{P})=\sum z_{\alpha_{\beta}} \ldots d y_{\alpha} d y_{\beta} \ldots=\sum \bar{z}_{\Upsilon \delta} \ldots d \bar{y}_{\Upsilon} d \bar{y}_{\delta} \ldots$, where $\bar{z}_{r \delta} \ldots, \bar{y}_{r}, \bar{y}_{\delta}, \ldots$ are also contained in the specialization ring of $\mathbf{Q}$ in $k(\mathbf{P})$, then $\sum z_{\alpha \beta}^{\prime} \ldots d y_{\alpha}^{\prime} d y_{\beta}^{\prime} \ldots=\omega^{\prime}(\mathbf{Q})$ and $\sum z_{r \delta}^{\prime} \ldots d \bar{y}_{\gamma}^{\prime} d \bar{y}_{\delta}^{\prime} \ldots=\bar{\omega}^{\prime}(\mathbf{Q})$ are identical. If the dimension of $\mathbf{V}<n-1$, then there exists a variety $\mathbf{W}^{n-1}$ which is algebraic over $k$ such that $\mathbf{U} \supset \mathbf{W} \supset \mathbf{V}$. Let $\mathbf{P}^{\prime}$ be a generic point of $\mathbf{W}$ over $k$. If $z$ is contained in the specialization ring of $\mathbf{Q}$ in $k(\mathbf{P})$, it is also contained in the specialization ring of $\mathbf{P}^{\prime}$ in $k(\mathbf{P})$. Further if $z^{*}$ is the specialization of $z$

8) Even if 10 is of the first kind, this is not always true. 
over $\mathbf{P} \rightarrow \mathbf{P}^{\prime}$ with respect to $k$, then the specialization of $z^{*}$ over $\mathbf{P}^{\prime} \rightarrow \mathbf{Q}$ with respect to $\bar{k}$ is identical with the specialization $z^{\prime}$ of $z$ over $\mathbf{P} \rightarrow \mathbf{Q}$ with respect to $k$. Therefore we can assume without loss of generality that the dimension of $\mathbf{V}$ is $n-1$. Let $\mathbf{U}^{*}$ be the normalization of $\mathbf{U}$ over $k$; let $\mathbf{P}^{*}$ be the corresponding generic point of $\mathbf{P}$, and let $\mathbf{Q}^{*}$ be a corresponding point of $\mathbf{Q}$ under the natural birational transformation between $\mathbf{U}$ and $\mathbf{U}^{*}$. Then $\mathbf{Q}^{*}$ is a simple point of $\mathbf{U}^{*}$ and $k\left(\mathbf{Q}^{*}\right)$ is an algebraic extension over $k(\mathbf{Q})$. Let $\omega^{*}$ be a differential form on $\mathbf{U}^{*}$ defined by $\omega^{*}\left(\mathbf{P}^{*}\right)=\omega(\mathbf{P})$; then since $\mathbf{Q}^{*}$ is simple $\omega^{* \prime}\left(\mathbf{Q}^{*}\right)$ $=\sum z_{\alpha \beta}^{\prime} \ldots d y_{\alpha}^{\prime} d y_{\beta}^{\prime} \ldots$ and $\bar{\omega}^{* \prime}\left(\mathbf{Q}^{*}\right)=\sum \bar{z}_{\gamma \delta}^{\prime} \ldots d \bar{y}_{\gamma}^{\prime} d \bar{y}_{\delta}^{\prime} \ldots$ are identical. If $\left(t_{1}, \ldots\right.$, $\left.t_{n-1}\right)$ is a set of elements of $k(\mathbf{Q})$ such that $k(\mathbf{Q}) / k\left(t_{1}, \ldots, t_{n-1}\right)$ is (separably) algebraic, then $\omega^{\prime}(\mathbf{Q})-\bar{\omega}^{\prime}(\mathbf{Q})$ is expressed in one and only one way as a polynomial of $d t_{i}(i=1, \ldots, n-1)$ :

$$
\omega^{\prime}(\mathbf{Q})-\bar{\omega}^{\prime}(\mathbf{Q})=\sum w_{i j} \ldots d t_{i} d t_{j} \ldots .
$$

Then we have $\omega^{* \prime \prime}\left(\mathbf{Q}^{*}\right)-\bar{\omega}^{* \prime}\left(\mathbf{Q}^{*}\right)=\sum w_{i j} \ldots d t_{i} d t_{j} \ldots$. As $k\left(\mathbf{Q}^{*}\right) / k(\mathbf{Q})$ is (separably) algebraic, $k\left(\mathbf{Q}^{*}\right) / k\left(t_{1}, \ldots, t_{n-1}\right)$ is also (separably) algebraic, and hence $w_{i j} \ldots$, ect. must be equal to 0 , because $\omega^{* \prime}\left(\mathbf{Q}^{*}\right)=\bar{\omega}^{* \prime}\left(\mathbf{Q}^{*}\right)$. Therefore $\omega^{\prime}(\mathbf{Q})$ $=\bar{\omega}^{\prime}(\mathbf{Q})$.

THEOREM 4. Assumptions being as in the above theorem, let $\omega$ be of the first kind. Then $\omega^{\prime}$ is also of the first kind.

Proof. We use the same notations as in the proof of the preceeding theorem. We may also assume without loss of generality that $\mathbf{V}$ is of dimension $n-1$. As $\mathbf{Q}^{*}$ is simple on $\mathbf{U}^{*}, \omega^{* \prime}$ is of the first kind on the locus of $\mathbf{Q}^{*}$ over $k$ in $\mathbf{U}^{*}$. Therefore the proof may by reduced to the following lemma.

Lemma 3. Suppose that $k\left(\mathbf{Q}^{*}\right)$ is an algebraic extension over $k(\mathbf{Q})$ and $\omega^{*}\left(\mathbf{Q}^{*}\right)=\omega(\mathbf{Q})$. If $\omega^{*}\left(\mathbf{Q}^{*}\right)$ is of the first kina, then $\omega(\mathbf{Q})$ is also of the first kind.

Proof. If we suppose that this is not true, there must exist a prime divisor $\mathfrak{F}$ of $k(\mathbf{Q})$ such that $\omega(\mathbf{Q})$ is not finite at $\mathfrak{P}$. Let $t_{1}, \ldots, t_{n-1}$ be a set of uniformizing parameters at $\mathfrak{B}$ in $k(\mathbf{Q})$. Let $\mathfrak{S}^{*}$ be a prime divisor of $k\left(\mathbf{Q}^{*}\right)$ which is an extension of $\mathfrak{P}$ and let $\left(t_{1}^{*}, \ldots, t_{n-1}^{*}\right)$ be a set of uniformizing parameters at $\mathfrak{P}^{*}$ in $k\left(\mathbf{Q}^{*}\right)$. Suppose $\mathfrak{P}^{* e} \|_{\mathfrak{P}}$. As $\omega(\mathbf{Q})$ is not finite at $\mathfrak{P}$, we can assume that

$$
\omega^{*}\left(\mathbf{Q}^{*}\right)=\omega(\mathbf{Q})=a d t_{1} \ldots d t_{s}+\ldots,
$$

where $a$ is an element in $k(\mathbf{Q})$ and $\nu_{\mathfrak{B}}(a)<0$. Since $\omega^{*}$ is of the first kind, $\omega^{*}\left(\mathbf{Q}^{*}\right)$ is finite at $\mathfrak{P}^{*}$ and $\theta\left(\mathbf{Q}^{*}\right)=d t_{s+1} \ldots d t_{n-1}$ is finite at $\mathfrak{B}^{*}$; therefore $\theta_{1}\left(\mathbf{Q}^{*}\right)$ $=\omega^{*}\left(\mathbf{Q}^{*}\right) \cdot \theta\left(\mathbf{Q}^{*}\right)=a d t_{1} \ldots d t_{s} d t_{s+1} \ldots d t_{n-1}$ is also finite at $\mathfrak{B}^{*}$. But as $d t_{1} \ldots$ $d t_{n-1}=b d t_{1}^{*} \ldots d t_{n-1}^{*}$, where $b$ is an element of $k\left(\mathbf{Q}^{*}\right)$ and $\nu_{\mathfrak{B}^{*}}(b)=e-1, \theta_{1}\left(\mathbf{Q}^{*}\right)$ $=a b d t_{1}^{*} \ldots d t_{n-1}^{*}$, where $\nu_{\mathfrak{\beta}^{*}}(a b) \leqq-e+(e-1)<0$. This contradicts to the fact that $\theta_{1}\left(\mathbf{Q}^{*}\right)$ is finite at $\mathfrak{P}^{*}$. 
An example

In the case of characteristic $p \neq 0$, theorem 4 does not hold in general. Let $k$ be an algebraically closed field of characteristic $p$ and let $V^{1}$ be the variety defined over $k$ by $F\left(X_{1}, X_{2}\right)=X_{2}^{q}+X_{2}-X_{1}^{m}$, where $q=p^{r}, r>0, m>1, q+1$ $=m n$. Let $\left(x_{1}, x_{2}\right)$ be a generic point of $V$ over $k$. Then $d x_{1}$ is a differential of the first kind in $k\left(x_{1}, x_{2}\right)$. This is the example of F. K. Schmidt. ${ }^{9)}$ Let $t$ be a quantity such that $t$ and $k\left(x_{1}, x_{2}\right)$ are independent over $k$. Put $x_{1}=x, t x_{2}=y$, $P=(1, x, y, t)$. Then $k\left(x_{1}, x_{2}, t\right)=k(P)$. Let $U^{2}$ be the locus of $P$ over $k$ and consider a projective variety $\mathbf{U}^{2}$ which has a representative $U_{0}^{2}=U^{2}$ and let $\mathbf{P}$ be a gneric point of $\mathbf{U}$ with the representative $P_{0}=P$; let $\omega$ be the differential form defined on $\mathbf{U}$ by $\omega(\mathbf{P})=d x$. Then $\omega$ is the differential form of the first kind. However if $\mathbf{Q}$ is a point of $\mathbf{U}$ which has the representative $Q_{0}=(1, x, 0,0)$ and if $\mathbf{W}$ is the locus of $\mathbf{Q}$ over $k$, then the induced differential form $\omega^{\prime}$ by $\omega$ on $\mathbf{W}$ cannot be of the first kind.

Mathematical Institute, Nagoga University

9) F. K. Schmidt, Zur arithmetischen Theorie der algebraischen Funktionen II, §5. Math. Zeitschrift, Bd. 35 (1939). 